
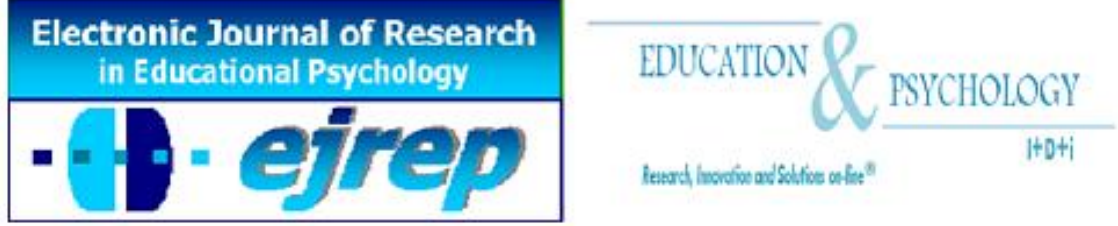

\title{
Aplicación de Estrategias Docentes para la Mejora de la Motivación Situacional del Alumnado en Educación Física
}

Francisco J. Almolda Tomás ${ }^{1}$, Javier Sevil Serrano ${ }^{1}$, José A. Julián Clemente ${ }^{2}$, Alberto Abarca Sos ${ }^{3}$, Alberto Aibar Solana $^{2}$ y Luis García González ${ }^{1}$.

${ }^{1}$ Departamento de Expresión Musical, Plástica y Corporal, Facultad de Ciencias de la Salud y del Deporte, Universidad de Zaragoza, Huesca.

${ }^{2}$ Departamento de Expresión Musical, Plástica y Corporal, Facultad de Ciencias Humanas y de la Educación, Universidad de Zaragoza, Huesca.

${ }^{3}$ Departamento de Expresión Musical, Plástica y Corporal, Facultad de Ciencias Sociales y Humanas, Universidad de Zaragoza, Teruel.

\section{España}

Correspondencia: Luis García González. Facultad de Ciencias de la Salud y del Deporte. Pza. Universidad, 3. CP 22001; Huesca (Spain). E-mail: 1garciag@unizar.es

(c) Education \& Psychology I+D+i and Editorial EOS (Spain) 


\section{Resumen}

Introducción. La Educación Física (EF) es un medio para favorecer la práctica deportiva en los adolescentes, y el profesor puede favorecer esta adherencia a la práctica generando motivación en el alumnado a través de su intervención docente. El objetivo del estudio fue desarrollar y aplicar estrategias de intervención docente para generar un clima motivacional óptimo y evaluar su incidencia sobre distintas variables que afectan a la motivación situacional de los alumnos en las clases de Educación Física, tomando como referencia la Teoría de las Metas de Logro y la Teoría de la Autodeterminación.

Método. La intervención estuvo basada en las áreas TARGET, desarrollándose una unidad didáctica de orientación deportiva donde participaron 113 alumnos de $3^{\circ}$ de ESO (58 chicos y 53 chicas $(M$ edad $=14.83, \mathrm{DT}=0.72)$ distribuidos en grupo experimental $(\mathrm{n}=46)$ y grupo control $(n=67)$. Los instrumentos utilizados midieron la percepción del clima motivacional, las necesidades psicológicas básicas, la motivación situacional autodeteminada y las consecuencias afectivas (satisfacción-diversión y aburrimiento).

Resultados. Los resultados mostraron que el grupo experimental obtuvo, respecto al grupo control, valores significativamente superiores en el clima motivacional maestría, y también en la autonomía, la percepción de competencia, la regulación identificada y la satisfaccióndiversión. Los valores de desmotivación fueron significativamente inferiores en el grupo experimental.

Discusión. Los resultados destacan la importancia de que el docente de EF transmita climas motivacionales tarea para optimizar diferentes variables que afectan a la motivación en las clases de EF, mejorando con ello el proceso de enseñanza-aprendizaje.

Conclusión. Destacamos que las áreas TARGET resultan efectivas para optimizar la intervención docente en el contenido de orientación deportiva y contribuyen a mejorar la motivación del alumnado en EF.

Palabras Clave: intervención docente; áreas TARGET; motivación autodeterminada; Educación Física; clima motivacional; mediadores psicológicos. 


\title{
Application of teaching strategies for improving students' situational motivation in Physical Education
}

\begin{abstract}
Introduction. Physical Education (PE) is a way to promote sports in adolescents, and the teacher can encourage this practice adherence to generating motivation in students through their educational intervention. The aim of the study was to develop and implement intervention strategies to generate optimal motivational climate and assessing their impact on variables related to situational motivation of student in PE lessons, under Goal Achievement Theory and Self-Determination Theory approach.
\end{abstract}

Method. The intervention was based on the TARGET areas, developing a orienteering sport teaching unit for 4 double sessions in which 113 students of 3rd E.S.O. participated (58 boys and 53 girls aged between 14 and $17 \mathrm{MD}=14.83 \mathrm{SD}=0.718$ ) divided into experimental group $(n=46)$ and control group $(n=67)$. Different instruments were used to assess motivational climate, perceived competence, autonomy and relatedness, self-determined motivation and affective consequences such as enjoyment-satisfaction and boredom.

Results. The results showed that the intervention was effective, because the experimental group showed significantly higher values than the control group in mastery motivational climate, autonomy, perceived competence, identified regulation and enjoyment. Expermiental group showed significantly lower values than the control group in amotivation.

Discussion. Results are discussed emphasizing the importance of the PE teacher, transmitting task-oriented motivational climates, and optimizing variables related to motivation in PE lesson, thereby imporving the teaching-learning process.

Conclusion. Therefore, it was proved that strategies based on TARGET areas that had been used, are effective for orienteering sport and constitute a good tool for encouraging a mastery climate of PE lessons, which is in turn an important element to optimizing students' motivational variables.

Keywords: didactic intervention; TARGET areas; self-determined motivation; Physical Education; motivational climate; psychological mediators. 


\section{Introducción}

Las experiencias de los adolescentes dentro de la Educación Física (EF), en los clubs deportivos o en la práctica de actividad física (AF) en su tiempo libre pueden ser cruciales para el desarrollo de patrones más adaptativos en la práctica futura (McKenzie, 2007). Dentro del ámbito educativo es considerado que el éxito de los programas de AF en EF escolar dependen en gran medida de la motivación experimentada por los alumnos, la cual puede ser manipulada en cierta medida por los docentes y su forma de impartir los contenidos (Cecchini, Fernández-Rio y Méndez-Giménez, 2014; Moreno-Murcia, Cervelló, González-Cutre, Julián y Del Villar, 2011). Por ello las actividades físicas tendrán consecuencias positivas tanto en EF escolar como en el deporte cuando los alumnos se encuentren motivados y experimenten resultados positivos cognitivos y/o afectivos debido a su participación (Gråstén, Jaakkola, Liukkonen, Watt y Yli-Piipari, 2012). Dos de las principales teorías socio-cognitivas que aportan los constructos teóricos para conocer el funcionamiento motivacional de los alumnos en las clases de EF son la Teoría de Metas de Logro (Nicholls, 1989) y la Teoría de la Autodeterminación (Ryan y Deci, 2002).

En el ámbito de la EF, la Teoría de Metas de Logro (Nicholls, 1989) ha contribuido a comprender los patrones cognitivos, emocionales y conductuales que se desarrollan en las clases (Papaioannou, Ampatzoglou, Kalogiannis y Sagovits, 2008). Su principal aportación es el concepto de clima motivacional (Ames, 1992) que se define como el conjunto de señales implícitas y/o explicitas, percibidas en el entorno, a través de las cuales se determinan las claves del éxito y el fracaso. Según las señales utilizadas se distinguen dos tipos de climas motivacionales: clima motivacional que implica a la tarea o clima maestría y clima motivacional que implica al ego o clima ejecución (Harwood, Spray y Keegan, 2008). El clima motivacional que implica a la tarea promueve el aprendizaje cooperativo, la elección de las tareas y su dominio y la evaluación de los alumnos basada en la mejora personal y autoreferenciada (Cervelló, Moreno, Martínez, Ferriz y Moya, 2011). Por el contrario, el clima motivacional que implica al ego promueve la competición interpersonal, limita la elección en los sujetos y utiliza la comparación social y pública en la evaluación (Braithwaite, Spray y Warburton, 2011). Asimismo, la percepción por parte de los alumnos de un clima maestría se asocia a conductas adaptativas como las metas del alumno centradas en la tarea, un mayor esfuerzo, disfrute e intención de practicar deporte (Amorose, Anderson-Butcher, Flesch y Klinefelter, 2005; Cecchini, González, Carmona y Contreras, 2004; Moreno, Cervelló y González-Cutre, 
2007) mientras que la percepción de un clima ejecución provoca conductas desadaptativas como un mayor aburrimiento, preocupación por el resultado final, sentimientos afectivos y negativos de presión (Balaguer, Duda, Castillo, Moreno y Crespo, 2009; Lochbaum, Stevenson y Hilario, 2009). Igualmente, otros estudios han mostrado cómo realmente los climas orientados hacia el ego o el rendimiento no generan únicamente consecuencias desadaptativas, si estos están combinados con climas orientados hacia el aprendizaje, ofreciendo una visión multidimensional de la motivación en el ámbito educativo, y destacando que los alumnos tienen capacidad de cambio o adaptación del tipo de meta en función del entorno o de sus necesidades (para un mayor desarrollo de la Teoría Revisada de la Orientación de Meta, ver De la Fuente, 2004).

La Teoría de la Autodeterminación (Ryan y Deci, 2002) establece la motivación como un continuo donde aparecen diferentes niveles de autodeterminación. Así encontramos de más a menos autodeterminada la motivación intrínseca, la motivación extrínseca y la desmotivación. La motivación intrínseca supone el compromiso de un sujeto con una actividad por el disfrute que le produce, siendo esta actividad un fin en sí misma. Dentro de la motivación extrínseca, de mayor a menor autodeterminación, encontramos diferentes formas de regulación: integrada, identificada, introyectada y externa, obedeciendo todas ellas a factores externos al individuo. Por último la desmotivación se caracteriza por la ausencia de intención, por parte del sujeto, de realizar algo (Deci y Ryan 2002). En el ámbito educativo, que los alumnos desarrollen un tipo u otro de motivación vendrá determinado por la satisfacción de sus necesidades psicológicas básicas (NPB), las cuales son propias de todos los seres humanos y explican las conductas de los individuos. Estos mediadores psicológicos son: la necesidad de autonomía, comprendiendo los esfuerzos de las personas por ser el origen de sus acciones y determinar su propio comportamiento; la necesidad de competencia, basada en experimentar eficacia en las acciones que realiza; y la necesidad de relación social, que hace referencia al esfuerzo por relacionarse con otros sujetos y experimentar satisfacción con el mundo social. La satisfacción de estas tres NPB se asocia con formas de motivación más autodeterminadas, mientras que su no satisfacción estará asociada con formas de motivación menos autodeterminadas (Deci y Ryan, 2000). A su vez, las diferentes formas de motivación, pueden desencadenar diversas consecuencias en los alumnos (Vallerand, 1997), las cuales se han clasificado como cognitivas (e.g., concentración, atención y memoria), afectivas (e.g., diversión, satisfacción y aburrimiento) y comportamentales (e.g., persistencia en la tarea, rendimiento). Por ello, en la medida que los profesores de EF realicen una intervención docente que contribuya 
a la satisfacción de estas tres NPB, el alumnado podrá experimentar formas de motivación más autodeterminadas, generando consecuencias más positivas a nivel afectivo, cognitivo y de conducta (Julián, 2012; Tessier, Sarrazin y Ntoumanis, 2010).

Asimismo, estas dos teorías motivacionales están apoyadas en el modelo jerárquico de Vallerand $(2001,2007)$ que sostiene la existencia de tres niveles jerárquicos (i.e., situacional, contextual y global). En el ámbito educativo, el nivel situacional hace referencia a las sesiones o a una unidad didáctica (UD), el nivel contextual engloba la EF en general y finalmente el nivel global está vinculado a un estilo de vida activo. Esto significa que la mejora de variables motivacionales a nivel situacional puede tener importantes consecuencias en los dos niveles siguientes debido a que la motivación experimentada en un nivel puede influir en el inmediatamente superior. Por tanto, el docente de EF se configura como un agente promotor de la práctica de AF presente y futura (Moreno, Huéscar y Cervelló, 2012).

Son numerosos los estudios en el ámbito educativo de la EF que han tratado de relacionar ambas teorías motivacionales (Moreno, Hellín, Hellín, Cervelló y Sicilia, 2008; Liukkonen, Barkoukis, Watt y Jaakkola, 2010), donde han sido estudiadas las relaciones entre el clima motivacional percibido por los alumnos y la satisfacción de sus NPB, observándose como, el fomento de un clima maestría se relaciona con mayores niveles de satisfacción de las tres NPB y el incremento a su vez de las formas de motivación más autodeterminadas (Moreno y Llamas, 2007). De forma contraria, el clima motivacional ejecución o que implica al ego se ha asociado con la desmotivación (Ommundsen y Eikanger-Kvalo, 2007).

En este sentido, el profesor de EF adquiere un papel fundamental, ya que es uno de los principales agentes modificadores del clima motivacional de clase y que puede proporcionar un clima motivacional óptimo (Julián, 2012). De esta manera, siguiendo los trabajos de Ames (1992) y Epstein (1989) los docentes cuentan con una serie de directrices con el fin de manipular las señales del entorno y fomentar en sus clases un clima motivacional maestría. Estas directrices se concretan en seis áreas denominadas con el acrónimo TARGET para referirse a las dimensiones de tarea (i.e., diseño de las tareas y actividades), autoridad (i.e., participación del sujeto en el proceso instruccional), reconocimiento (i.e., razones para el reconocimiento, distribución de recompensas), agrupación (i.e., forma y frecuencia en la que los sujetos interactúan), evaluación (i.e., establecimiento de estándares y criterios de evaluación) y tiempo (i.e., posibilidades de flexibilidad de la programación). En el ámbito educativo de la EF en el 
contexto español, existen estudios de intervención realizados a partir de las áreas TARGET que han demostrado su eficacia para conseguir un mayor clima tarea (e.g., Cecchini et al., 2001; González-Cutre, Sicilia y Moreno, 2011; Viciana, Cervelló, Ramírez-Lechuga, 2007). También existen programas mediante la implementación de las áreas TARGET en el contexto deportivo que han demostrado una gran eficacia, mostrando diferencias significativas en favor del clima tarea, y manteniendo los cambios de forma estable en el tiempo (Cecchini, Fernandez-Rio, Mendez-Gimenez, Cecchini y Martins, 2014).

De igual modo, en el contexto internacional son numerosos los estudios en las clases de EF que han estudiado los efectos de manipulación del clima tarea sobre diferentes variables motivacionales y consecuencias adaptativas (e.g., Bowler, 2009; Jaakkola y Liukkonen, 2006; Morgan, Kingston y Sproule, 2005; Wang, Liu, Chatzisarantis y Lim, 2010). Como muestra un reciente meta-análisis realizado sobre la efectividad de la intervenciones mediante el uso de las áreas TARGET en el contexto de EF, encontramos numerosos estudios que avalan este tipo de intervenciones y sus efectos a nivel comportamental, cognitivo y afectivo en el alumnado (Braithwaite et al., 2011), aunque la mayoría de estos estudios han evaluado sus efectos fundamentalmente en el nivel contextual de la motivación (i.e., EF en general), sin adentrarse en evaluar sus resultados en el nivel situacional (i.e., distintas UD dentro de la EF).

\section{Objetivos e hipótesis}

Tomando como sustento la literatura científica mencionada anteriormente, el estudio tiene un doble objetivo. En primer lugar, comprobar la eficacia de una intervención diferenciada a través del uso de estrategias motivacionales basadas en las áreas TARGET aplicadas a nivel situacional durante el contenido de orientación deportiva. Como segundo objetivo, planteamos evaluar el efecto de este tipo de intervención diferenciada sobre otras variables motivacionales a nivel situacional (i.e., NPB, motivación autodeterminada y consecuencias afectivas). Por ello, se plantearon dos hipótesis relacionadas con los objetivos expuestos anteriormente. En primer lugar, planteamos que una intervención docente basada en el uso de estrategias relacionadas con las áreas TARGET reflejaría un mayor clima motivacional maestría en las clases de EF durante el contenido de orientación deportiva. Como segunda hipótesis del estudio planteamos que al generar un mayor clima motivacional maestría se producirá una mayor satisfacción de las NPB, mayores niveles de motivación autodeterminada y una mayor satisfacción y diversión en el alumnado. 


\section{Método}

\section{Participantes}

En el estudio participaron 113 alumnos de EF (58 chicos y 53 chicas) con edades comprendidas entre los 14 y 17 años $(\mathrm{M}$ edad $=14.83$, DT $=0.72)$ pertenecientes a 5 grupos de $3^{\circ}$ de Educación Secundaria Obligatoria (ESO) de un centro público. Dos grupos conformaron el grupo experimental con un total de 46 alumnos $(\mathrm{M}$ edad $=14.83$, DT $=0.77)$ y los tres grupos restantes conformaron el control con un total de 67 alumnos $(\mathrm{M}$ edad $=14.84$, DT $=0.68)$.

\section{Instrumentos}

Escala de Percepción del Clima Motivacional (EPCM). La percepción del clima motivacional situacional en las clases de EF fue medida a través de “L'Echelle de Perception du Climat Motivational" (EPCM) de Biddle y col. (1995), en su traducción y adaptación española de Gutiérrez, Ruiz y López (2011). Se utilizaron 16 ítems de los 19 ítems que componen esta escala teniendo en cuenta cuatro factores de primer orden: Búsqueda de Progreso por los Alumnos, Promoción de Aprendizaje por el Profesor, Búsqueda de Comparación por los Alumnos y Miedo a Cometer Errores, agrupados, a su vez, en dos factores de segundo orden: 9 ítems para el Clima de Aprendizaje o Maestría, compuesto por los dos primeros factores de primer orden (e.g., "Los alumnos aprenden cosas nuevas y se sienten satisfechos", "El profesor se siente satisfecho cuando cada alumno aprende algo nuevo") y 7 ítems para el Clima de Comparación o Ejecución, compuesto por los dos últimos factores de primer orden (e.g., "Los alumnos intentan hacerlo mejor que los demás", "El profesor sólo se ocupa de quienes hacen bien los ejercicios"). El cuestionario estaba encabezado por la frase "En mis clases de la unidad didáctica de orientación...", seguida de los distintos ítems. El quinto factor que compone el cuestionario original (Promoción de comparación por el profesor) fue eliminado del estudio por la baja fiabilidad obtenida (Alpha de Cronbach $=.43$ ). Se obtuvieron valores alpha de Cronbach de .73 para la búsqueda de progreso por los alumnos, de .82 para la promoción de aprendizaje por el profesor, de .74 para la búsqueda de comparación por los alumnos y de .85 para el miedo a cometer errores. En los dos factores de segundo orden, el clima tarea obtuvo valores de alpha de Cronbach de .84 y el clima ego de .69 respectivamente. 
Escala de Medición de las Necesidades Psicológicas Básicas (BPNES). Se utilizó la versión traducida al castellano (Moreno, González-Cutre, Chillón y Parra, 2008) de la Escala de Medición de las Necesidades Psicológicas Básicas (BPNES; Vlachopoulos y Michailidou (2006). Para evaluar las NPB a nivel situacional, este instrumento estaba precedido de la frase "En las clases de la unidad didáctica de orientación..." seguida de 12 ítems que miden la percepción de autonomía (4 ítems; e.g., “Tengo la oportunidad de elegir cómo realizar los ejercicios"), percepción de competencia (4 ítems; e.g., "Siento que he tenido una gran progresión con respecto al objetivo final que me he propuesto") y percepción de relaciones sociales (4 ítems; e.g., "Me siento muy cómodo/a cuando hago los ejercicios con los/las demás compañeros/as"). Se obtuvieron valores alfa de Cronbach de .66 para la percepción de autonomía, de .69 para la percepción de competencia y .77 para la percepción de relaciones con los demás.

Escala de Motivación Situacional (SIMS-14). Se adaptó para la EF a nivel situacional para un contenido de orientación deportiva el cuestionario validado en contexto educativo español (Martín-Albo, Nuñez y Navarro, 2009) del original (Guay, Vallerand, y Blanchard, 2000). El SIMS-14 fue utilizado para evaluar la motivación situacional en alumnos de EF para el contenido de orientación deportiva. Está compuesta por 14 ítems agrupados en cuatro factores: motivación intrínseca (4 ítems; e.g., "Porque creo que era interesante"), regulación identificada ( 3 ítems; e.g., "Lo he hecho por mi propio bien"), regulación externa (3 ítems; e.g., "Porque se supone que lo tenía que hacer") y desmotivación (4 ítems; e.g., "Debe haber buenas razones para haber realizado orientación, pero personalmente no veo ninguna"). Los participantes respondían a la pregunta “¿Por qué has participado este año en la unidad de orientación?”. Debido a que esta versión no ha sido utilizada en el contexto de una UD de orientación en EF, se analizó la validez del instrumento a través de un análisis factorial confirmatorio (AFC), testando el mismo modelo teórico presentado en la validación española de Martín-Albo y col. (2009) y comparándolo con los datos obtenidos en este estudio. Los resultados del AFC indicaron un ajuste adecuado de los datos para la estructura de los cuatro factores descrita anteriormente, a través de los distintos índices de ajuste evaluados $\left(\chi^{2}\right.$ (71, $\left.\mathrm{N}=113)=119.31, \mathrm{p}<.001 ; \chi^{2} / g . l .=1.68 ; \mathrm{RMSEA}=.08 ; \mathrm{SRMR}=.07 ; \mathrm{CFI}=.94 ; \mathrm{TLI}=.92\right)$. Los coeficientes alpha de Cronbach revelaron valores de fiabilidad de .87 para la motivación intrínseca, de .73 para la regulación identificada, de .72 para la regulación externa y de .84 para la desmotivación. 
Escala de diversión de los sujetos con la práctica deportiva (CDPD). Se utilizó la validación española en EF (Cecchini y col., 2004) del original (Duda y Nicholls, 1992). Este cuestionario mide la diversión situacional que tienen los sujetos con la práctica de la UD evaluada. El cuestionario consta de 8 ítems agrupados en dos factores denominados aburrimiento (3 ítems; e.g., "Normalmente me aburría en las clases de orientación") y satisfaccióndiversión (5 ítems; e.g., "Me solía divertir haciendo orientación”). La pregunta inicial del cuestionario es “¿Cómo te lo has pasado en las clases de orientación de este curso?”. El análisis de fiabilidad obtuvo valores de alpha de Cronbach de .90 para la satisfacción-diversión y de .86 para el aburrimiento.

Las respuestas a los diferentes ítems de todos los cuestionarios fueron reflejadas en una escala tipo Likert con un rango de respuesta de 1 a 5, donde 1 correspondía a totalmente en desacuerdo y 5 a totalmente de acuerdo. En el caso de las variables que han presentado una fiabilidad inferior a .70 (clima ego con valores de .69, percepción de autonomía con valores de .66 y percepción de competencia con valores de .69) se pueden considerar aceptables debido el pequeño número de ítems que componen el factor (Hair, Anderson, Tatham y Black, 1998; Nunnally y Bernstein, 1995).

\section{Procedimiento}

En primer lugar, se contactó con los directores de este centro educativo para explicarles los objetivos del estudio y su consentimiento para impartir una de las unidades didácticas de la programación anual didáctica del docente de EF así como para la administración de diferentes cuestionarios.

De forma previa a la intervención docente, el profesor del grupo experimental fue instruido específicamente para transmitir de forma adecuada un clima maestría a través de distintas estrategias de intervención. La formación estuvo enfocada en el conocimiento y la forma de aplicación en el contexto de la EF escolar de las teorías motivacionales de Metas de Logro y Autodeterminación tal y como se ha expuesto anteriormente. La intervención del docente en el grupo experimental se llevó a cabo a través de la aplicación de una serie de estrategias basadas en las seis áreas del TARGET (tarea, autoridad, reconocimiento, agrupación, evaluación y tiempo) establecidas por Ames (1992) con apoyo de las directrices de Julián y Pinos (2011) para el contenido de orientación. Una vez finalizada la UD se administraron en el aula los diferentes cuestionarios, tanto en el grupo control como en el grupo experimental, con la pre- 
sencia del investigador principal y con la ausencia de su profesor de EF. De forma previa a la cumplimentación de los cuestionarios, fueron indicadas una serie de instrucciones para rellenar los mismos, se les animó a contestar de la manera más sincera posible ya que eran cuestionarios anónimos que no iban a formar parte de la evaluación de la UD. El tiempo empleado por los estudiantes para cumplimentarlos fue de 15 a 20 minutos.

\section{Intervención en el grupo experimental}

De forma resumida, a continuación se presentan las estrategias utilizadas durante la UD en el grupo experimental. En relación a la dimensión tarea, se diseñaron actividades basadas en la variedad, respetando las posibilidades y limitaciones de los alumnos. Se buscó el reto personal a través de una progresión en las actividades, estableciendo diferentes niveles de práctica. Por último, siempre al comienzo de la sesiones y de las actividades se destacaron cuáles eran los objetivos a conseguir.

Respecto a la dimensión autoridad, se implicó a los sujetos en el proceso de toma de decisiones permitiéndoles tomar decisiones sobre ciertos aspectos del proceso de enseñanzaaprendizaje como la colocación las balizas, el tiempo para su búsqueda o el lugar de su colocación. Se trató de fomentar el autocontrol y autodirección en los alumnos, estableciéndose un plazo determinado para conseguir descubrir todas las balizas, de manera que fuesen regulándose para conseguirlo en el tiempo establecido. Se proporcionó un feedback descriptivo (comentándole al alumno cómo había realizado la acción), explicativo (analizado las causas de sus fallos) e interrogativo (conduciendo a la reflexión del alumnado para que alcanzará los conocimientos específicos de la actividad). Además se proporcionó feedback a cada alumno, al menos una vez durante cada sesión.

Asimismo, en la dimensión agrupación se fomentaron las relaciones sociales entre el alumnado mediante el planteamiento de actividades en parejas o pequeños grupos. Además las agrupaciones fueron flexibles y heterogéneas, con pequeñas variaciones durante las actividades de una misma sesión y a lo largo de todas las sesiones.

En relación, a la evaluación se diseñó una evaluación diagnóstica que permitió al profesor establecer las diferentes situaciones motrices, teniendo en cuenta las posibilidades y limitaciones respecto al contenido. Se implicó al alumnado en el proceso de evaluación dándole la posibilidad de elección de los porcentajes de calificación de los criterios a evaluar, 
respetando unos criterios mínimos establecidos por el profesor. El proceso de evaluación fue privado y significativo comunicando de forma personal e individual los resultados a cada alumno. Se valoró en gran medida el progreso y el dominio personal respecto a la evaluación inicial.

Por último, se posibilitó el tiempo suficiente para desarrollar las diferentes actividades, respetando el ritmo propio de aprendizaje de cada alumno y grupo de trabajo. De esta forma, el alumnado tuvo la oportunidad de consolidar los diferentes conocimientos específicos y de aumentar su percepción de competencia.

\section{Intervención en el grupo control}

Por otro lado, el profesor que impartió la UD en el grupo control no recibió ninguna instrucción y desarrolló sus clases sin tener en cuenta la intención de transmitir un clima maestría. Siguiendo la categorización antes desarrollada de las áreas TARGET, se pudo observar que en cuanto a la al área tarea no siempre había variedad en las tareas, repitiendo alguna de ellas en diferentes sesiones. Un aspecto importante es que las tareas no eran adaptables ni permitían diferentes niveles de dificultad, siendo este nivel común para todo el grupo. El docente no transmitió los objetivos a alcanzar a sus alumnos y únicamente lo hizo al comienzo de la sesión, para dar a conocer los objetivos generales.

En cuanto a la dimensión autoridad el docente dejaba cerradas todas las tareas, utilizando un estilo docente más controlador, impidiendo así que los alumnos tomaran ningún tipo de decisión ni que desarrollaran su autonomía. Debido a que no se establecían diferentes niveles de dificultad, los alumnos no tenían las mismas posibilidades de reconocimiento y sólo eran reconocidos aquellos discentes que conseguían superar las tareas. Además el feedback ofrecido por el profesor en muy pocas ocasiones referenciada al proceso y progreso individual, centrándose este en la mayoría de los casos en el resultado final, sin fomentar así las reflexiones del alumnado. Las agrupaciones siempre eran libres y a elección del alumnado, lo que suponía que estos siempre trabajaban con los mismos compañeros durante todas las sesiones y siempre en grupos reducidos de dos o tres alumnos.

En cuanto a la dimensión evaluación, se observó que no hubo evaluación diagnóstica además de que siempre el profesor comentaba los resultados de forma pública y ante el resto de los compañeros. La evaluación en alguna ocasión tenía criterios referidos al progreso pero 
en su mayoría se centraba en el resultado obtenido. El sistema de evaluación fue determinado por el profesor y en ningún momento tuvo en cuenta la opinión del alumnado.

Por último, en referencia a la dimensión tiempo, cuando se cumplía la hora de fin de clase las tareas se terminaban, pero estas no se retoman en sesiones posteriores, aunque hubieran quedado incompletas.

\section{Diseño y análisis de datos}

Se utilizó un diseño cuasi-experimental con grupo control no equivalente (Campbell y Stanley, 1966), ya que al realizar la investigación en un ámbito educativo real, los grupos de clases ya están establecidos por el centro de enseñanza y no se puede respetar la aleatorización.

La variable independiente fue el desarrollo de una UD dentro de la cual se aplicaron diferentes estrategias motivacionales en el grupo experimental. Por otro lado, las variables dependientes fueron el clima motivacional percibido por el alumno (clima tarea o maestría y clima ego o rendimiento), las NPB (autonomía, percepción de competencia y relaciones sociales), la motivación situacional autodeterminada (motivación intrínseca, regulación identificada, regulación externa y desmotivación) y las consecuencias afectivas (satisfacción-diversión y aburrimiento). Ninguno de los alumnos tenía un conocimiento previo sobre el contenido que iba a impartirse. Al tratarse del nivel situacional de la motivación, entendido como una UD al completo, no se pudo realizar una medición pre-test, ya que todas las variables estudiadas hacían referencia de forma específica al contenido de orientación deportiva, por lo que no se encontraba coherente medir las distintas variables motivacionales sobre un contenido que no conocía el alumnado. Además, el diseño del estudio plantea dos partes diferenciadas: una primera parte que realiza la evaluación de la efectividad de las estrategias desarrolladas a través de las posibles diferencias encontradas sobre el clima motivacional percibido por el alumnado; y una segunda parte donde se evaluarán las consecuencias que estas diferencias en el clima motivacional pueden ejercer sobre otras variables motivacionales a nivel situacional, como las NPB, la motivación autodeterminada, la satisfacción-diversión y el aburrimiento.

Cada grupo tuvo un profesor diferente, ambos con experiencia en el área de Educación Física. En el grupo experimental el profesor realizó una intervención específica aplicando estrategias para transmitir de forma eficaz un clima motivacional que implicaba a la tarea. 
Esta intervención vino precedida de una formación específica con un total de 60 horas sobre las teorías motivacionales utilizadas en el estudio y además una aplicación práctica sobre el diseño de tareas y situaciones de aprendizaje bajo las premisas de estas teorías y que fueron específicamente desarrolladas para aplicarlas en el contenido de orientación deportiva. Por otro lado, el profesor del grupo control no tuvo ningún tipo de formación ni tampoco conocía ni utilizaba de forma específica y consciente las estrategias de intervención relacionadas con las áreas TARGET, por lo que en el grupo control no hubo ningún tipo de manipulación, y el otro profesor utilizó una metodología tradicional. Se puede comprobar de forma más específica las diferencias en la intervención de ambos profesores en el apartado de Procedimiento desarrollado anteriormente. El planteamiento de las UD está basado en el curriculum oficial vigente (Orden de 9 de mayo de 2007, BOA de 1 de junio de 2007). La intervención fue realizada durante un total de 4 sesiones dobles de 1 hora y 40 minutos y con una frecuencia de una sesión semanal.

Para el análisis de datos, se realizó inicialmente un análisis de normalidad que indicó la pertinencia de utilizar estadística paramétrica. En primer lugar se realizó un análisis de fiabilidad para los distintos factores que componen los instrumentos, mediante el Alpha de Cronbach. Los resultados descriptivos fueron calculados a través de la media y la desviación típica. Para el análisis de diferencias se utilizó un MANOVA de un factor (Grupo). También se calculó el tamaño del efecto $\left(\eta_{\mathrm{p}}{ }^{2}\right)$ para evaluar la magnitud de las diferencias ya que elimina el efecto del tamaño de la muestra. En los distintos análisis realizados se utilizó el programa estadístico SPSS 19.0.

\section{Resultados}

El MANOVA realizado muestra un efecto principal del factor Grupo (Lambda de Wilks $\left.=.784 ; F(11,101)=2.523 ; p=.007 ; \eta_{p}{ }^{2}=.216\right)$. Para observar en primer lugar la efectividad de la intervención, se analizó el clima motivacional percibido por los alumnos a nivel situacional. Puede observarse en la Tabla 1 que se encontraron diferencias significativas en el factor de primer orden búsqueda de progreso por los alumnos $(F(1,111)=5.59, p=.020)$ y en el factor de segundo orden clima maestría $(F(1,111)=5.68, p=.019)$, con valores superiores en el grupo experimental. 
Tabla 1. Estadísticos Descriptivos Para Cada Grupo y Comparación por Pares en el Análisis de Diferencias en el Clima Motivacional Situacional.

\begin{tabular}{|c|c|c|c|c|c|c|c|c|c|c|c|}
\hline \multirow[b]{2}{*}{ Variables } & \multicolumn{2}{|c|}{$\begin{array}{c}\text { Grupo } \\
\text { experimental }\end{array}$} & \multicolumn{2}{|c|}{$\begin{array}{l}\text { Grupo } \\
\text { control }\end{array}$} & \multirow[b]{2}{*}{$\begin{array}{l}\text { Dif. } \\
\text { medias }\end{array}$} & \multirow[b]{2}{*}{$\begin{array}{l}\text { Error } \\
\text { típico }\end{array}$} & \multirow{2}{*}{$\mathrm{F}_{(1,111)}$} & \multirow[b]{2}{*}{$p$} & \multirow{2}{*}{$\eta_{\mathrm{p}}^{2}$} & \multicolumn{2}{|c|}{ IC $95 \%$ diferencias } \\
\hline & $\mathrm{M}$ & DT & $\mathrm{M}$ & DT & & & & & & $\begin{array}{l}\text { Límite } \\
\text { inferior }\end{array}$ & $\begin{array}{l}\text { Límite } \\
\text { superior }\end{array}$ \\
\hline Clima Maestría & 4.01 & $(0.67)$ & 3.74 & $(0.53)$ & .270 & .113 & 5.68 & .019 & .049 & .046 & .494 \\
\hline Búsqueda Progreso por Alumnos & 3.66 & $(0.61)$ & 3.39 & $(0.56)$ & .265 & .112 & 5.59 & .020 & .048 & .043 & .488 \\
\hline Promoción aprendizaje Profesor & 4.36 & $(0.83)$ & 4.09 & $(0.67)$ & .275 & .141 & 3.77 & .055 & .033 & -.006 & .555 \\
\hline Clima Ejecución & 3.19 & $(0.49)$ & 3.09 & $(0.63)$ & .091 & .111 & 0.67 & .414 & .006 & -.129 & .312 \\
\hline Búsqueda Comparación por Alumnos & 3.97 & $(0.87)$ & 3.74 & $(0.70)$ & .230 & .149 & 2.37 & .126 & .021 & -.066 & .525 \\
\hline Miedo a Cometer Errores & 2.60 & $(0.86)$ & 2.61 & $(0.83)$ & -.012 & .162 & 0.01 & .939 & .001 & -.333 & .308 \\
\hline
\end{tabular}

Posteriormente, para evaluar el efecto de esta intervención en el resto de variables motivacionales situacionales se evaluaron también las diferencias existentes entre ambos grupo en las NPB, motivación autodeterminada y consecuencias afectivas (Tabla 2). En este caso, en las necesidades psicológicas básicas se encontraron diferencias significativas en las variables autonomía $(F(1,111)=11.78, p=.001)$ y percepción de competencia $(F(1,111)=16.06, p<.001)$, con valores superiores en el grupo experimental. En cuanto a las variables sobre la motivación autodeterminada se encontraron diferencias significativas en la regulación identificada $(F(1,111)=6.65, p=.011)$, con valores superiores en el grupo experimental, y en la Desmotivación $(F(1,111)=5.83, p=.017)$, con valores significativamente inferiores en el grupo experimental. Por último, con relación a las consecuencias, la variable satisfacción-diversión muestra valores significativamente superiores en el grupo experimental $(F(1,111)=5.84, p=.017)$. 
Tabla 2. Estadísticos Descriptivos Para Cada Grupo y Comparación por Pares en el Análisis de Diferencias en las NPB, Motivación Autodeterminada y Consecuencias Afectivas

\begin{tabular}{|c|c|c|c|c|c|c|c|c|c|c|c|}
\hline \multirow[b]{2}{*}{ Variables } & \multicolumn{2}{|c|}{$\begin{array}{c}\text { Grupo } \\
\text { experimental }\end{array}$} & \multicolumn{2}{|c|}{$\begin{array}{l}\text { Grupo } \\
\text { control } \\
\end{array}$} & \multirow[b]{2}{*}{$\begin{array}{c}\text { Dif. } \\
\text { medias }\end{array}$} & \multirow[b]{2}{*}{$\begin{array}{l}\text { Error } \\
\text { típico }\end{array}$} & \multirow{2}{*}{$\mathrm{F}_{(1,111)}$} & \multirow[b]{2}{*}{$p$} & \multirow{2}{*}{$\eta_{\mathrm{p}}^{2}$} & \multicolumn{2}{|c|}{ IC 95\% diferencias } \\
\hline & M & DT & M & DT & & & & & & $\begin{array}{l}\text { Límite } \\
\text { inferior }\end{array}$ & $\begin{array}{l}\text { Límite } \\
\text { superior }\end{array}$ \\
\hline Autonomía & 3.30 & $(0.74)$ & 2.85 & $(0.64)$ & .454 & .132 & 11.78 & .001 & .096 & .192 & .715 \\
\hline Competencia & 3.77 & $(0.66)$ & 3.28 & $(0.61)$ & .486 & .121 & 16.06 & $<.001$ & .126 & .246 & .727 \\
\hline Relación con los demás & 3.95 & $(0.80)$ & 3.74 & $(0.73)$ & .209 & .146 & 2.05 & .155 & .018 & -.080 & .497 \\
\hline Motivación intrínseca & 3.27 & $(0.84)$ & 2.94 & $(0.98)$ & .322 & .179 & 3.25 & .074 & .028 & -.032 & 677 \\
\hline Regulación identificada & 3.30 & $(0.87)$ & 2.88 & $(0.80)$ & .412 & .160 & 6.65 & .011 & .057 & .095 & .728 \\
\hline Regulación externa & 3.82 & $(0.88)$ & 3.62 & $(0.87)$ & .197 & .168 & 1.37 & .245 & .012 & -.137 & .531 \\
\hline Desmotivación & 2.39 & $(0.92)$ & 2.84 & $(1.00)$ & -.450 & .186 & 5.83 & .017 & .050 & -.819 & -.081 \\
\hline Satisfacción - Diversión & 3.50 & $(0.88)$ & 3.06 & $(0.98)$ & .437 & .181 & 5.83 & .017 & .050 & .079 & .796 \\
\hline Aburrimiento & 2.40 & $(0.99)$ & 2.81 & $(1.15)$ & -.407 & .209 & 3.08 & .054 & .033 & -.821 & .007 \\
\hline
\end{tabular}

\section{Discusión}

Este estudio presentaba dos objetivos fundamentales. En primer lugar comprobar la eficacia de una intervención docente a nivel situacional, basada en las áreas TARGET en el contenido de orientación deportiva, y en segundo lugar evaluar su efecto sobre otras variables motivacionales como las NPB, la motivación autodeterminada y las consecuencias afectivas. Con relación a la primera hipótesis, donde se planteó que una intervención docente basada en las áreas TARGET reflejaría un mayor clima motivación maestría en las clases de EF durante el contenido de orientación deportiva, podemos afirmar que parece confirmarse. 
Tras el análisis de resultados, encontramos que el grupo experimental en el que fueron aplicadas las estrategias motivacionales obtuvo una mayor percepción de clima maestría a nivel situacional (i.e., UD de orientación deportiva). En esta misma línea, otros estudios (Barkoukis, Tsorbatzoudis y Grouios, 2008; Cecchini et al., 2014; Cuevas, Contreras y GarcíaCalvo, 2012; Morgan y Kingston, 2008) han mostrado la utilidad de manipular las áreas TARGET (Ames, 1992) para conseguir un clima motivacional óptimo, el cual resultará fundamental para optimizar las variables motivacionales en EF y lograr consecuencias más adaptativas. Además, la literatura científica ha indicado a través de distintos estudios de intervención que el uso de las áreas TARGET no solamente permitirá generar un clima motivacional tarea en momentos determinados si no que también permitirán obtener efectos permanentes a lo largo del tiempo (Digelidis, Papaioannou, Laparidis, y Christodoulidis, 2003; Valentini, y Rudisill, 2004). Además, siguiendo con el modelo jerárquico de Vallerand (2001, 2007) los efectos encontrados a nivel situacional (i.e., UD) podrán tener influencia en el nivel contextual (i.e., EF en general) por lo resalta la importancia de realizar intervenciones específicas para cada UD ya que ayudarán a configurar los procesos motivacionales en niveles superiores. Sin embargo, apenas se han realizado estudios de intervención donde se hayan medido los efectos dentro del nivel situacional de la motivación en EF (Braithwaite et al., 2011), si bien en este estudio encontramos que la eficacia sobre el clima motivacional generado a nivel situacional en el contenido de orientación deportiva es importante, por lo que el desarrollo de estrategias específicas de intervención para cada uno de los contenidos abordados en EF será fundamental.

Como segunda hipótesis del estudio planteamos anteriormente que un mayor clima motivacional maestría produciría una mayor satisfacción de las NPB, mayores niveles de motivación autodeterminada y una mayor satisfacción y diversión en el alumnado, hipótesis que podemos confirmar. El mayor nivel de clima maestría percibido por el alumnado del grupo experimental, objetivo de la intervención docente presente, tiene una serie de repercusiones sobre las variables motivacionales que establece la Teoría de la Autodeterminación. De esta forma, esta intervención docente, al generar un mayor clima motivacional orientado a la tarea o maestría ha producido una mayor satisfacción de las necesidades psicológicas básicas de autonomía y percepción de competencia, en línea con estudios anteriores (e.g., Cox y Williams, 2008; Standage et al., 2006). 
Por otro lado, el fomento de un clima maestría y la satisfacción de las NPB, han dado lugar, en el grupo experimental, a valores significativamente superiores en los perfiles de motivación más autodeterminados y valores significativamente inferiores en la desmotivación de los alumnos. Estos resultados muestran consonancia con los encontrados en el estudio de González-Cutre y colaboradores (2011) donde el estudio de intervención que llevo a cabo supuso además de un aumento del clima motivacional tarea una mayor motivación intrínseca. Los resultados obtenidos se muestran en la línea de otras investigaciones en EF (Moreno, Conte, Hellín, Vera y Cervelló, 2008; Moreno, Jiménez, Gil, Aspano y Torrero, 2011; Sproule, Wang, Morgan, McNeill, y McMorris, 2007) donde se mostraba que el clima motivacional maestría se relacionaba de forma positiva y significativa con las formas de motivación más autodeterminadas. De igual modo, otros autores (Moreno, González-Cutre y Ruiz, 2009; Taylor y Ntoumanis, 2007) han mostrado como aquellos alumnos que presentan una mayor motivación autodeterminada en las clases de EF, son aquellos que sienten en mayor medida satisfechas sus NPB, del mismo modo que ocurre en la presente investigación.

Asimismo, aunque los alumnos han experimentado una mayor satisfacción de sus NPB, obteniendo mayores niveles de motivación autodeterminada, se puede observar cómo estos alumnos no han alcanzado niveles significativamente más altos de motivación intrínseca, obteniendo únicamente un nivel significativamente más elevado en la regulación identificada, la cual es considerada uno de los perfiles más autodeterminados (Haerens, Vereecken, Maes, Bourdeaudhuij, 2010). Esto coincide con el estudio de Spray (2002), el cual señala la dificultad de desarrollar una motivación intrínseca en la EF ya que es una asignatura obligatoria donde el alumno participa en las clases porque tiene que hacerlo, ya que es lo que determina la institución educativa.

Finalmente y en relación a las consecuencias afectivas estudiadas (satisfaccióndiversión y aburrimiento), el grupo experimental, con valores significativamente más altos en formas de motivación autodeterminada, muestra una mayor percepción de satisfaccióndiversión que el grupo control. De forma análoga, el estudio de intervención de Wallhead y Ntoumanis (2004) mostró en el grupo experimental un aumento de la diversión en las clases de EF. Estos resultados se muestran en la línea de otros estudios anteriores centrados en las consecuencias afectivas donde se observa una fuerte relación directa entre la motivación autodeterminada y la diversión (Moreno, Hernández y González-Cutre, 2009; Ryan y Deci, 2007; Taylor, Ntoumanis y Smith, 2009). Por ello es importante que los alumnos desarrollen 
perfiles de motivación autodeterminada que den lugar a mayores sensaciones de diversión, satisfacción y/o disfrute durante las clases de EF, ya que esto puede contribuir a la adherencia a la práctica de AF y deportiva por parte de los alumnos en su tiempo de ocio (Vallerand, 2007; Yli-Piipari, Leskinen, Jaakkola y Liukkonen, 2012).

De forma general los resultados del estudio son congruentes con los obtenidos por Papaioannou, Tsigilis y Kosmidou (2007) donde un clima motivacional maestría daba como resultado una mayor motivación autodeterminada, regulada a través de una mayor satisfacción de las NPB, y dando lugar a unas consecuencias afectivas relacionadas con una mayor diversión durante las clases de EF.

Por ello, lo expuesto hasta el momento reafirma los postulados que relacionan la Teoría de Metas de Logro y la Teoría de la Autodeterminación (Ciani, Sheldon, Hilpert y Easter, 2011), y que en nuestro estudio se ha llevado a cabo dentro del nivel situacional (i.e., unidad didáctica de orientación deportiva). En este sentido, los antecedentes sociales, entre los que se encuentra el clima motivacional generado por el docente, influyen sobre las NPB y por consiguiente sobre el nivel de motivación, siendo esta más o menos autodeterminada, generando todo ello una serie de consecuencias a nivel afectivo como la satisfacción-diversión en las clases de EF para este contenido específico. En base a estos resultados, debemos resaltar que los efectos generados en la motivación a nivel situacional podrán, sucesivamente, tener efectos en la motivación contextual (i.e., la educación física, en general) y finalmente, en la motivación global (i.e., adoptando un estilo de vida saludable). Por tanto, resulta fundamental el fomento de un clima motivacional maestría durante las clases de EF, ya que este contribuirá a optimizar las variables motivacionales de los alumnos, como la motivación situacional, y también podrá generar conductas más adaptativas, relacionadas con hábitos de vida saludables y práctica continuada de AF en el tiempo libre (Jaakkola, Washington y Yli-Piipari, 2013; Yli-Piipari et al., 2012).

A pesar de los resultados obtenidos, el presente estudio cuenta con una serie de limitaciones que es necesario destacar. En primer lugar, el diseño del estudio no permite realizar un pre-test al tratarse de una evaluación dentro del nivel situacional de la motivación, donde el alumnado no tenía experiencias previas. Sin embargo, sería posible utilizar medidas contextuales sobre la EF que permitirían aumentar el control de posibles variables extrañas que puedan influir sobre los resultados. Igualmente, podrían utilizarse otras medidas previas en dife- 
rentes UD, para comprobar si esas diferencias encontradas se manifiestan también en otros contenidos sobre los que no ha habido una intervención específicamente orientada a generar un clima tarea.

Así, se ha evidenciado cómo las áreas TARGET (Ames, 1992), son un recurso útil y eficaz del que disponen los docentes de EF, para diseñar y aplicar estrategias motivacionales que fomenten un clima maestría en el contenido de orientación deportiva. El presente estudio se ha ocupado de una parte mínima de la amplia y compleja relación de variables motivacionales presentes en EF, además de centrarse en un único contenido de las múltiples opciones que se presentan en el curriculum oficial vigente en la Orden de 9 de mayo de 2007 (BOA de 1 de junio de 2007; Aragón, España). Por ello se establece como futura línea de estudio ver cómo pueden afectar otras variables que no han sido recogidas en el estudio (e.g., el soporte de autonomía, la orientación disposicional o los hábitos de práctica de AF) y evaluar el efecto de intervenciones a nivel situacional sobre variables a nivel contextual o global. Además, sería interesante desarrollar estrategias que puedan aplicarse en otros contenidos o ámbitos de las actividades físico-deportivas, ya que según señalan Cervelló, Iglesias, Moreno, Jiménez y Del Villar (2004) los docentes de EF suelen mostrar un estilo estable de determinación del clima motivacional en los diferentes contenidos de EF, lo que sugiere la necesidad de desarrollar programas de formación para conseguir que los docentes generen un clima motivacional óptimo en las clases de EF. Por último, otro aspecto que puede optimizar este tipo de investigaciones sería la utilización de soporte digital y metodología observacional para poder establecer diferencias detalladas sobre la aplicación de estrategias, así como la frecuencia e intensidad de las mismas, y reorientar la intervención docente de una forma más eficaz. 


\section{Referencias}

Ames, C. (1992). Achievement goals, motivational climate, and motivational processes. En G. C. Roberts (Ed.), Motivation in sport and exercise (pp. 161-176). Champaign, IL: Human Kinetics.

Amorose, A. J., Anderson-Butcher, D., Flesch, S., y Klinefelter, L. (2005). Perceived motivational climate and self-determined motivation in male and female high school athletes. Research Quarterly for Exercise and Sport, 76, 96-97.

Balaguer, I., Duda, J. L., Castillo, I. Moreno, Y., y Crespo, M. (2009). Interacciones entre las perspectivas situacionales y disposicionales de meta y el burnout psicológico de los tenistas júnior de la elite internacional. Acción Psicológica, 6(2), 63-75.

Barkoukis, V., Tsorbatzoudis, H., y Grouis, G. (2008). Manipulation of motivational climate in physical education: effects of a seven-month intervention. European Physical Education Review, 4, 367-387. DOI: 10.1177/1356336X08095671

Biddle, S., Cury, F., Goudas, M., Sarrazin, P., Famose, J. P., y Durand, M. (1995). Development of scales to measure perceived physical education class climate: A cross national project. British Journal of Education Psychology, 65, 341-358. DOI: 10.1111/j.20448279.1995.tb01154.x

Bowler, M. (2009, Septiembre). The influence of the TARGET motivational climate structures on pupil physical activity levels during year 9 athletics lessons. Comunicación presentada en la British Educational Research Association Annual Conference, Manchester, Inglaterra. Resumen extraido de http://www.leeds.ac.uk/educol/documents/184297.pdf.

Braithwaite, R., Spray, C. M., y Warburton, V. E. (2011). Motivational climate interventions in physical education: A meta-analysis. Psychology of Sport and Exercise, 12(6), 628638. DOI: 10.1016/j.psychsport.2011.06.005

Campbell, D. T., y Stanley, J. (1966). Experimental and quasi-experimental designs for research. Boston: Houghton Mifflin Co.

Cecchini, J. A., Fernandez-Rio, J., Mendez-Gimenez, A. (2014). Effects of Epstein's TARGET on adolescents' intentions to be physically active and leisure-time physical activity. Health Educational Research, 29(3), 485-490. DOI: 10.1093/her/cyu007. 
Cecchini, J. A., Fernandez-Rio, J., Mendez-Gimenez, A., Cecchini, C., y Martins, L. (2014). Epstein's TARGET framework and motivational climate in sport: effects of a field-based, long-term intervention program. International Journal of Sports Science and Coaching, 9.

Cecchini, J. A., Gonzalez, C., Carmona, A., Arruza, J., Escarti, A., y Balague, G. (2001). The influence of the physical education teacher on intrinsic motivation, selfconfidence, anxiety, and pre- and post-competition mood states. European Journal of Sport Science, 1, 111 .

Cecchini, J. A., González, C., Carmona, A. M., y Contreras, O. (2004). Relaciones entre clima motivacional, la orientación de meta, la motivación intrínseca, la auto-confianza, la ansiedad y el estado de ánimo en jóvenes deportistas. Psicothema, 16(1), 104-109.

Cervelló, E. M., Iglesias, D., Moreno, P., Jiménez, R., y Del Villar, F. (2004). Aplicación de modelos de ecuaciones estructurales al estudio de la motivación de los alumnos en las clases de educación física. Revista de Educación, 335, 371-382.

Cervelló, E. M., Moreno, J. A., Martínez, C., Ferriz, R., y Moya, M. (2011). El papel del clima motivacional, la relación con los demás, y la orientación de metas en la predicción del flow disposicional en educación física. Revista de Psicología del Deporte, 20(1), 165178.

Ciani, K. D., Sheldon, K. M., Hilpert, J. C., y Easter, M. A. (2011). Antecedents and trajectories of achievement goals: A self-determination theory perspective. British Journal of Educational Psychology, 81(2), 1-21. DOI: 10.1348/000709910X517399

Cox, A., y Williams, L. (2008). The roles of perceived teacher support, motivational climate, and psychological need satisfaction in students' physical education motivation. Journal of Sport and Exercise Psychology, 30(2), 222-239.

Cuevas, R., Contreras, O., y García-Calvo, T. (2012). Effects of an experimental program to improve the motivation in physical education of Spanish students. Social and Behavioral Sciences, 47, 734-738. DOI: 10.1016/j.sbspro.2012.06.726

De la Fuente, J. (2004). Perspectivas recientes en el estudio de la motivación: la Teoría de la Orientación de Meta. Electronic Journal of Reseach in Educatinal Psychology, 2(1), 3562. 
Deci, E. L. y Ryan, R. M. (2000). The «what» and «why» of goal pursuits: Human needs and the self-determination of behaviour. Psychological Inquiry, 11, 227-268.

Deci, E.L. y Ryan, R.M. (2002). Handbook of Self-determination Research. Rochester: The University of Rochester Press.

Digelidis, N., Papaioannou, A., Laparidis, K., y Christodoulidis, T. (2003). A one-year intervention in 7th grade physical education classes aiming to change motivational climate and attitudes toward exercise. Psychology of Sport and Exercise, 4(3), 195-210. DOI: 10.1016/S1469-0292(02)00002-X

Duda, J. L., y Nicholls, J. G. (1992). Dimensions of achievement motivation in scholwork and sport. Journal of Educational Psichology, 84(3), 290-299. DOI: 10.1037//00220663.84.3.290

Epstein, J. (1989). Family structures and student motivation: A developmental perspective. En C. Ames y R. Ames (Eds.) Research on motivation in education (pp. 259-295). Academic Press, New York.

González-Cutre, D., Sicilia, A., y Moreno, J. A. (2011). Un estudio cuasi-experimental de los efectos del clima motivador tarea en las clases de Educación Física. Revista de Educación, 356, 677-700. DOI: 10-4438/1988-592X-RE-2010-356-056

Gråstén, A., Jaakkola, T., Liukkonen, J., Watt, A., y Yli-Piipari, S. (2012). Prediction of enjoyment in school physical education. Journal of Sports Science and Medicine, 11, 260269.

Guay, F., Vallerand, R. J., y Blanchard, C. (2000). On the assessment of state intrinsic and extrinsic motivation: The situational motivation scale (SIMS). Motivation and Emotion, 24(3), 175-213.

Gutiérrez, M, Ruiz, L. M., y López, E. (2011). Clima motivacional en Educación Física: concordancia entre las percepciones de los alumnos y las de sus profesores. Revista de Psicología del Deporte, 2(20), 312-335.

Haerens, L., Vereecken, C., Maes, L., y De Bourdeaudhuij, I. (2010). Relationship of physical activity and dietary habits with body mass index in the transition from childhood to adolescence: a 4-year longitudinal study. Public Health Nutrition, 13(10), 1722-1728. DOI: $10.1017 / \mathrm{S} 1368980010002284$ 
Hair, J. F., Anderson, R. E., Tatham, R. L., y Black, W.C. (1998). Multivariate Data Analysis. Upper Saddle River, New Jersey: Prentice-Hall.

Harwood, C., Spray, C. M., y Keegan, R. (2008). Achievement goal theories in sport. In T. Horn (Ed.), Advances in sport psychology (pp. 157-186). Champaign, IL: Human Kinetics.

Jaakkola, T., y Liukkonen, J. (2006). Changes in students' self-determined motivation and goal orientation as a result of motivational climate intervention within high school physical education classes. International Journal of Sport and Exercise Psychology, Special Issue: Sport Psychology in the School Environment: An International Perspective, 4, 302-324. DOI: 10.1080/1612197X.2006.9671800

Jaakkola, T., Washington, T., y Yli-Piipari, S. (2013). The association between motivation in school physical education and self-reported physical activity during Finnish junior high school: The self-determination theory approach. European Physical Education Review 19, 1-15. DOI: $10.1177 / 1356336 X 12465514$

Julián, J. A. (2012). Motivación e intervención docente en la clase de educación física. Tándem. Didáctica de la Educación Física, 40, 7-17.

Julián, J. A., y Pinos, M. (2011). Ejemplificación de educación física para segundo ciclo de primaria. Actividad: Orientación. Zaragoza: Departamento de Educación, Universidad, Cultura y Deporte del Gobierno de Aragón.

Liukkonen, J., Barkoukis, V., Watt, A., y Jaakkola, T. (2010). Motivational climate and students' emotional experiences and effort in physical education. Journal of Educational Research, 103(5), 295-308. DOI: 10.1080/00220670903383044

Lochbaum, M., Stevenson, S., y Hilario, D. (2009). Achievement goals, thought about intense physical activity, and exerted effort: A mediational analysis. Journal of Sport Behavior, 32, 53-69.

Martín-Albo, J., Nuñez, J. L., y Navarro, J. (2009). Validation of the Spanish Version of the Situational Motivation Scale (EMSI) in the educational context. The Spanish Journal or Psychology, 12(2), 799-807.

McKenzie, T. L. (2007). The preparation of physical educators: A public health perspective', Quest, 59(4), 346-57. 
Moreno, B., Jiménez, R., Gil, A., Aspano, M. I., y Torrero, F. (2011). Análisis de la percepción del clima motivacional, necesidades psicológicas básicas, motivación autodeterminada y conductas de disciplina de estudiantes adolescentes en las clases de Educación Física, Motricidad. European Journal of Human Movement. 26, 1-24.

Moreno, J. A., Cervelló, E., y González-Cutre, D. (2007). Young athletes’ motivational profiles. Journal of Sports Science and Medicine, 6, 172-179.

Moreno, J. A., y Llamas, L. S. (2007). Predicción de la importancia concedida a la educación física según el clima motivacional y la motivación autodeterminada en estudiantes adolescentes. Enseñanza, 25, 137-155.

Moreno, J. A., Conte, L., Hellín, P., Vera, J. A., y Cervelló, E. (2008). Predicción de la motivación autodeterminada según las estrategias para mantener la disciplina y la orientación motivacional en estudiantes adolescentes de educación física. Apuntes de Psicología, 26(3), 501-516.

Moreno, J. A., González-Cutre, D., Chillón, M., y Parra, N. (2008). Adaptación a la educación física de la escala de las necesidades psicológicas básicas en el ejercicio. Revista Mexicana de Psicologia, 25(2), 295-303.

Moreno, J. A., Hellín, P., Hellín, G., Cervelló, E., y Sicilia, A. (2008). Assessment of motivation in spanish physical education students: Applying achievement goals and selfdetermination theories. The Open Education Journal, 1(1), 15-22. DOI: $10.2174 / 1874920800801010015$

Moreno, J. A., González-Cutre, D., y Ruiz, L. M. (2009). Self-determined motivation and physical education importance. Human Movement, 10(1), 5-11. DOI: 10.2478/v10038008-0022-7

Moreno, J. A., Hernández, A., y González-Cutre, D. (2009). Complementando la teoría de la autodeterminación con las metas sociales: un estudio sobre la diversión en educación física. Revista Mexicana de Psicología, 26(2), 213-222.

Moreno, J. A., Cervelló, E., González-Cutre, D., Julián, J. A., y Del Villar, F. (2011). La motivación en el deporte. Claves para el éxito. Barcelona: Inde.

Moreno, J. A., Huéscar, E., y Cervelló, E. (2012). Prediction of adolescents doing physical activity after completing secondary education. The Spanish Journal of Psychology, 15(1), 90-100. 
Morgan, K., Kingston, K., y Sproule, J. (2005). Effects of different teaching styles on the teacher behaviours that influence motivational climate and pupils' motivation in physical education. European Physical Education Review, 11(3), 257-285.

Morgan, K., y Kingston, K. (2008). Development of a self-observation mastery intervention programme for teacher education. Physical Education \& Sport Pedagogy, 13(2), 109129. DOI: $10.1080 / 17408980701345634$

Nicholls, J. (1989). The Competitive Ethos and Democratic Education. Cambridge, MA: Harvard University Press.

Nunnally, J. C., y Bernstein, I. J. (1995). Teoría psicométrica. Madrid: McGraw-Hill.

Ommundsen, Y., y Eikanger-Kvalo, S. (2007). Autonomy-Mastery, supportive or performance focused? Different teacher behaviours and pupils' outcomes in physical education. Scandinavian Journal of Educational Research, 51(4), 385-413.

Orden de 9 de mayo de 2007, del Departamento de Educación, Cultura y Deporte, por la que se aprueba el currículo de la Educación secundaria obligatoria y se autoriza su aplicación en los centros docentes de la Comunidad autónoma de Aragón. Boletín Oficial de Aragón de 1 de junio de 2007, 65, 8871-9024.

Papaioannou, A., Tsigilis, N., y Kosmidou, E. (2007). Measuring perceived motivational climate in physical education. Journal of Teaching in Physical Education, 26(3), 236-259.

Papaioannou, A., Ampatzoglou, G., Kalogiannis, P., y Sagovits, A. (2008). Social agents, achievement goals, satisfaction and academic achievement in youth sport. Psychology of Sport and Exercise, 9, 122-141. DOI: 10.1016/j.psychsport.2007.02.003

Ryan, R. M., y Deci, E. L. (2002). An overview of self-determination theory: An organismicdialectical perspective. En E. L. Deci, y R. M. Ryan (Eds.), Handbook of selfdetermination research (pp. 3-33). Rochester, NY: University of Rochester.

Ryan, R. M., y Deci, E. L. (2007). Active human nature: Self-determination theory and the promotion and maintenance of sport, exercise, and health. En M. S. Hagger, y N. L. Chatzisarantis (Eds). Intrinsic motivation and self-determination in exercise and sport (pp. 1-19). Champaign, IL: Human Kinetics.

Standage, M., Duda, J. L., y Ntoumanis, N. (2006). Students' motivational processes and their relationship to teacher ratings in school physical education: A self-determination theory approach. Research Quarterly for Exercise and Sport, 77(1), 100-110. 
Sproule, J., Wang, C. K. J, Morgan, K., McNeill, M., y McMorris, T. (2007). Effects of motivational climate in Singaporean physical education lessons on intrinsic motivation and physical activity intention. Personality and individual differences, 43, 1037-1049. DOI: 10.1016/j.paid.2007.02.017

Taylor, I., y Ntoumanis, N. (2007). Teacher motivational strategies and student selfdetermination in physical education. Journal of Educational Psychology, 99(4), 747-760. DOI: $10.1037 / 0022-0663.99 .4 .747$

Taylor, I., Ntoumanis, N., y Smith, B. (2009). The social context as a determinant of teacher motivational strategies in physical education. Psychology of Sport and Exercise, 10(2), 235-243. DOI: 10.1016/j.psychsport.2008.09.002

Valentini, N., y Rudisill, M. E. (2004). An inclusive mastery climate intervention and the motor skill development of children with and without disabilities. Adapted Physical Activity Quarterly, 21, 330-347.

Vallerand, R. J. (1997). Toward a hierarchical model of intrinsic and extrinsic motivation. En M. P. Zanna (Ed.), Advances in experimental social psychology (pp. 271-360). New York: Academic Press.

Vallerand, R. J. (2007). Intrinsic and Extrinsic Motivation in Sport and Physical Activity. A Review an a Look at the Future. En G. Tenenbaum, y R. C. Eklund (Eds.). Handbook of Sport Psychology ( $3^{\text {a }}$ ed., pp. 59-83). New York: John Wiley.

Viciana, J., Cervelló, E. M., y Ramírez-Lechuga, J. (2007). Effect of manipulating positive and negative feedback on goal orientations, perceived motivational climate, satisfaction, task choice, perception of ability, and attitude toward physical education lessons. Perceptual and Motor Skills, 105(1), 67-82. DOI: 10.2466/pms.105.1.67-82

Vlachopoulos, S. P., y Michailidou, S. (2006). Development and initial validation of a measure of autonomy, competence, and relatedness in exercise: The Basic Psychological Needs in Exercise Scale. Measurement in Physical Education and Exercise Science, 10, 179-201. DOI: 10.1207/s15327841mpee1003_4.

Wang, J. C. K., Liu, W. C., Chatzisarantis, N. L. D., y Lim, C. B. S. (2010). Influence of perceived motivational climate on achievement goals in physical education: A structural equation mixture modeling analysis. Journal of Sport and Exercise Psychology, 32(3), 324-338. 
Wallhead, T. L., y Ntoumanis, N. (2004). Effects of a sport education intervention on students' motivational responses in physical education. Journal of Teaching in Physical Education, 23, 4-18

Yli-Piipari, S., Watt, A., Jaakkola, T., Liukkonen, J., y Nurmi, J., (2009). Relationships between physical education students' motivational profiles, enjoyment, state anxiety, And self-reported physical activity. Journal of Sports Science and Medicine, 8, 327-336.

Yli-Piipari, S., Leskinen, E., Jaakkola, T., y Liukkonen, J. (2012). Predictive role of physical education motivation: The developmental trajectories of physical activity during grades 7-9. Research Quarterly for Exercise and Sport, 83(4), 560-570. DOI: $10.5641 / 027013612804582650$ 